\title{
Endovascular treatment of dysfunctional arteriovenous fistula in hemodialyzed patients the results of one year follow-up
}

\author{
Pawel Maga', Marek Krzanowski², Pawel Kaczmarczyk², Jolanta Koscielniak², \\ Lukasz Partyka ${ }^{2}$, Andrzej Belowski², Lukasz Drelicharz², Pawel Kuczia ${ }^{3}$, \\ Krzysztof Malinowski ${ }^{4}$, Rafal Nizankowski' \\ 'Department of Angiology, Jagiellonian University Medical College, Krakow, Poland \\ ${ }^{2}$ Angiomed, Medical Clinic, Krakow, Poland \\ ${ }^{3}$ Department of Medicine, Jagiellonian University Medical College, Krakow, Poland \\ ${ }^{4}$ Faculty of Health Sciences, Jagiellonian University Medical College, Krakow, Poland
}

\begin{abstract}
Introduction. The arteriovenous fistula (AVF) dysfunction is a common reason for vascular access problem in chronically hemodialyzed patients. It is caused by stenosis or occlusion located either in inflow artery, anastomosis or outflow vein. Revascularization of these pathologies can be achieved in surgical or endovascular (PTA) manner. The aim of this study was to evaluate both immediate and late endovascular treatment results of dysfunctional fistulas in chronically hemodialyzed patients.
\end{abstract}

Material and methods. We included in our observation 106 patients with end stage renal disease, who underwent PTA within arteriovenous fistulas. We used conventional and unified techniques of endovascular therapy. Procedural results were evaluated after I, 3, 6 and 12 months based on fistula sufficiency during hemodialysis. Results. In 96 (90.6\%) cases the initial result of PTA was good. We achieved improvement in blood flow through AVF and successful hemodialysis. In 10 cases (9.4\%) results were not satisfactory. None of our patients developed neither worsening in the blood flow through AVF nor compromised blood circulation distally to AVF. No serious complications (MI, stroke, death) occurred during procedure or hospital stay. After 12 months, in 52 patients AVF were functioning properly. In 20 cases, because of fistula dysfunction, reintervention was necessary (primary patency 66\%). Considering all patients, also these with successful reintervention, 69 AVF were functioning properly after 12 months (secondary patency $86 \%$ ).

Conclusions. To conclude, the immediate and long-term PTA outcomes of arteriovenous fistulas with currently available techniques and equipment are satisfying. PTA is a safe manner of prolonging patency rate of AVF in patients requiring permanent hemodialysis.

Key words: arteriovenous fistula, hemodialysis, percutaneous transluminal angioplasty, revascularization

Acta Angiol 2016; 22, 4: 143-149

Address for correspondence: Pawel Maga, Department of Angiology, Jagiellonian University Medical College, Skawinska 8, 3I-066 Krakow, Poland, tel.: +486025870 8I, e-mail: maga.pawel@gmail.com 


\section{Introduction}

While the chronic kidney disease and end-stage renal disease (ESRD) population is continuously expanding $[1,2]$ functional vascular access ensures an adequate and effective dialysis in this group of patients. Native arteriovenous fistulae (AVF) are the best and most durable access for hemodialysis [3]. Catheters, on the contrary, have high rates of infection and thrombosis and should be used when fistulae and grafts cannot be fashioned [4].

The AVF dysfunction is a common cause for vascular access problem in chronically hemodialyzed patients. It is due to stenosis or occlusion located in the inflow artery, anastomosis or outflow vein.

Access dysfunction may be caused by stenotic lesion as a result of neointimal hyperplasia at the surgical anastomosis [5] and may lead to thrombosis [6] and subsequent access failure. Such complications occur frequently, increasing patient's morbidity and creating additional costs for healthcare system.

Percutaneous transluminal angioplasty (PTA) is an accepted treatment for obstructive lesions. Although the rate of restenosis after endovascular intervention remains high [7], it appears to be the optimal treatment modality. Patency of a vessel after percutaneous angioplasty has been shown to be equivalent with surgical treatment though endovascular approach generates less complications $[8,9]$.

The purpose of our study was to determine the immediate and long-term clinical effects of PTA of dysfunctional native fistulae using currently available endovascular techniques.

\section{Material and methods}

A consecutive series of 106 ESRD patients treated with hemodialysis (HD) and requiring endovascular AVF repair were included in the study. They were referred to the Department of Angiology, Jagiellonian University Medical College, Krakow, Poland (JUMC) - tertiary, high volume interventional therapy center. Data were systematically collected, anonymized and analyzed independently for treatment outcomes. Study was planned and executed according to ethical 5 standards of local Ethics Committee. As all the data were fully anonymized before analysis, no formal consents and approvals were necessary, except for the routine informed consent for imaging and PTA according to hospital procedures.

\section{Population}

All patients were treated with maintenance HD 3 times a week. Hemodialysis vintage ranged between 8 and 81 months (mean 45.619 .0 months). There were
Table I. Demographic and clinical profiles of studied subjects

\begin{tabular}{|l|c|}
\hline Age (years) & n (\%) \\
\hline Males & 59.2 \\
\hline Coronary artery disease, including: & $47(43.9)$ \\
\hline Myocardial infarction & $20(18.7)$ \\
\hline $\begin{array}{l}\text { History of coronary artery } \\
\text { angioplasty }\end{array}$ & $16(14.9)$ \\
\hline History of CABG & $7(6.5)$ \\
\hline Atrial fibrillation & $4(3.7)$ \\
\hline Congestive heart failure & $19(17.8)$ \\
\hline History of stroke/TIA & $6(5.6)$ \\
\hline Hypertension & $79(73.8)$ \\
\hline Hemodialysis & $107(100)$ \\
\hline Diabetes & $40(37.4)$ \\
\hline On insulin & $34(31.8)$ \\
\hline On oral medication & $2(1.9)$ \\
\hline COPD & $9(8.4)$ \\
\hline Asthma & $2(1.9)$ \\
\hline Cigarette smoking & $10(9.3)$ \\
\hline History of cigarette smoking & $14(13.0)$ \\
\hline
\end{tabular}

Data are presented as numbers (and percentages). CABG — coronary artery bypass grafting; COPD — chronic obstructive pulmonary disease; TIA — transient ischemic attack

no cases of inadequate fistula maturation or concomitant central vein occlusion. In 69 referred patients AVF was a 'primary' access (first AVF, with no history of surgical reconstruction or previous endovascular procedures). Five patients experienced single surgical reconstruction before present referral, 23 - two reconstructions and in 10 patients 3 surgical reconstructions were performed. No previous endovascular procedures were performed in any analyzed AVF. Demographic and clinical profiles of study subjects are shown in Table I.

All referrals for intervention were based on the standard Kidney Disease Outcomes Quality Initiative (KDOQI) and the 'Fistula First' approach [10-12] as well as local standards at referring HD centers, which suggest intervention if one or more of the following occur: abnormalities on physical examination, poor dialyzer blood flow ( $<200 \mathrm{~mL} / \mathrm{min}$ ), elevated venous/arterial pressure detected on HD monitor, increased bleeding, inability to cannulate, or decrease in dialysis adequacy measured by dialysis index $\mathrm{Kt} / \mathrm{V}<\mathrm{I} .2$ [13] or URR $<65 \%$ [14]. Referral details are summarized in Table 2.

Functional Doppler ultrasound of AVF were performed in all patients before procedure in order to locate the stenotic segment (later confirmed in angiography) and to assess AVF anatomy for further, post-procedural monitoring. Significant stenosis was defined as a reduction in luminal diameter of more than $50 \%$ on access angiogram. 
Table 2. Referral details

\begin{tabular}{|l|c|}
\hline Poor dialyzer blood flow $(<200 \mathrm{~mL} / \mathrm{min})$ & $\mathbf{n ( \% )}$ \\
\hline $\begin{array}{l}\text { Elevated venous/arterial line pressure on } \\
\text { HD monitor }\end{array}$ & $87(81.3)$ \\
\hline $\begin{array}{l}\text { Increased bleeding and/or inability } \\
\text { to cannulate }\end{array}$ & $19(17.7)$ \\
\hline $\mathrm{Kt} / \mathrm{V}<\mathrm{I} .2$ & $91(85.0)$ \\
\hline $\mathrm{URR}<65 \%$ & $85(79.4)$ \\
\hline
\end{tabular}

$\mathrm{Kt} / \mathrm{V}$ - dialyzer clearance of urea multiplied by dialysis time and divided by volume distribution of urea; URR - urea reduction ratio

\section{AVF characteristics and technical procedure description}

All arteriovenous fistulae were native forearm or arm fistulae created as anastomoses between the side or end segments of radial or brachial artery with the adequate segments of cephalic or basilic vein. They were stenosed in $72(67.3 \%)$ patients and occluded/ /thrombosed in 35 (32.7\%) patients. Stenosis within the inflow artery was found in 24 (22.4\%) cases, within anastomosis in 52 (48.6\%) and in outflow vein in 101 cases $(94.4 \%)$ respectively.

In the group of patients with occluded fistulae, concomitant occlusion of inflow artery, anastomosis and outflow vein was observed in 15 cases, whereas occlusion of anastomosis and outflow vein in seven cases and outflow vein only - in thirteen.

Vascular access for procedure was established by cannulation of common femoral artery ( 90 patients $85 \%)$, brachial artery (7 patients - 6.6\%) or distal portion of fistula's outflow vein ( 9 patients $-8.4 \%$ ). Doppler ultrasound was done before all attempts to puncture femoral artery. In case of occlusion or significant stenosis of femoral, iliac arteries or in cases of chronic total occlusion of subclavian artery, which proved to be impossible to treat due to technical reasons, brachial artery or fistula was punctured. Classic Seldinger method was used. In cases of femoral access a $90 \mathrm{~cm}$ long 6Fr sheath (Brite Tip, Cordis, Miami Lakes, USA) was placed in proximal brachial or axillar artery. In the remaining patients, a standard $5 \mathrm{Fr} \mathrm{II} \mathrm{cm}$ long sheath was used. Once the vascular access was established, the $A V$ fistula was cannulated in a standard fashion, using 0.035 " (Radifocus guide wire M, Terumo, Leuven, Belgium) or 0,018" (V-I8 Control Wire, Boston Scientific, Galway, Ireland) hydrophilic wires and standard angiographic catheters.

All patients underwent prolonged POBA angioplasty (Plain Old Balloon Angioplasty) (3 minutes [IQR I.8$-3.5 \mathrm{~min}]$ ) of stenosed or thrombosed vessels. Standard balloon catheters with dimensions ranging from $3 \mathrm{~mm}$ to $6 \mathrm{~mm}$ (depending on respective vessel diameter) were used (Passeo, Biotronik, Bullach, Switzerland; Powerflex Pro, Cordis, Miami Lakes, USA; Armada, Abbott Vascular, Santa Clara, USA) (Fig. I). In 12 patients (II.2\%) isolated angioplasty was not satisfactory. In these few cases angioplasty with scoring balloon (AngioScore, Biotronik, Bulach, Switzerland) was performed with good angiographic result.

Thrombosed fistulae were treated using the same technique with hydrophilic guidewires and balloon catheters. Due to inadequate reimbursement in the Polish Health System, drug-eluting balloon angioplasty was not used. Stents or declotting devices have never been necessary in our experience to improve the final result. Moreover, due to the location of treated vessels and need for repetitive vessel punctures during hemodialises, the use of stents is controversial. For these reasons they were not utilized.

Procedural success was defined as an achievement of a satisfactory final residual diameter stenosis $(<30 \%)$ by

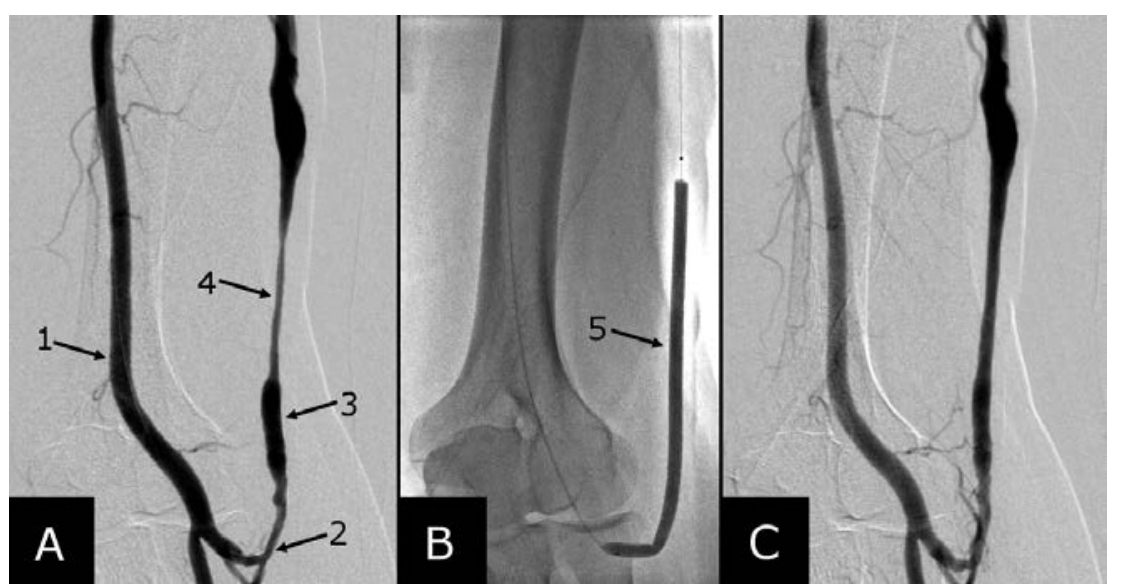

Figure I. Prolonged Plain Old Balloon Angioplasty of arteriovenous fistula. A. Pre PTA; B. PTA; C. Post PTA; I — inflow artery; 2 - anastomosis; 3 - outflow vein; 4 - stenosis; 5 - balloon catheter 
quantitative angiography at the end of the procedure (without flow-limiting dissection or hemodynamically significant gradient). Periprocedural treatment and monitoring were standardized. Site closure devices were used sporadically (AngioSeal, St. Jude Medical, Zaventem, Belgium). Puncture site was examined on the first day after procedure by Doppler ultrasound for any puncture site complications (arterio-venous fistula, pseudoaneurysm, dissection). Hemodialysis was performed on the day before and after the procedure in all patients. Limited evidence exists on the periprocedural administration of heparin and antiplatelet drugs. Based on available reports and center experience patients received $75 \mathrm{mg} /$ day of aspirin daily; in addition they received $40 \mathrm{mg} /$ day of enoxaparine for the first month after the procedure (HD - free days).

Fistula efficiency during HD was repeatedly verified the day after procedure and then on months I, 3, 6 and 12 post-procedure. Well-functioning AVF has been defined by means of quantitative parameters of dialysis adequacy, i.e.:

$-\mathrm{Kt} / \mathrm{V}>\mathrm{I} .2-\mathrm{I} .4$,

- URR < 68-70\% and

- dialyzer blood flow ranging between 250 and $400 \mathrm{~mL} /$ /min.

All criteria had to be met to confirm AVF function. Dysfunction of AVF without possibility for future successful revascularization or patient death were defined as treatment failure.

\section{Statistical analysis}

Statistical analysis was performed using SPSS package (SPSS Inc. Released 2009. PASW Statistics for Windows, Version 18.0. Chicago: SPSS Inc.). Variables were evaluated in descriptive statistics. Data were analyzed in respective contingency tables. Mann-Whitney- $U$ test and chi-squared test (with Yates correction if applicable) were used to assess differences. P-values less than 0.05 were considered as statistically significant. Kaplan-Meier estimator curve was generated using $R$ survival package (Therneau T [2014] A Package for Survival Analysis in S. R package version 2.37-7).

\section{Results}

As the monitoring pattern of dialysis parameters in referring centers was highly inconsistent, it was not possible to retrieve specific numerical data on $\mathrm{Kt} / \mathrm{V}$, URR, and dialyzer flow changes in many cases. Thus we decided to approach post procedural follow-up in a simplified way (see methods section). Meeting all 3 criteria combined with procedural success was considered a treatment success; it was achieved in 96 cases (90.6\%). In 4 patients (3.7\%) we were not able to pass through the occluded vein and in $6(5.6 \%)$ cases there was little or no improvement in blood flow through AVF despite of apparently successful PTA. None of our patients developed neither worsening in the blood flow through AVF nor compromised blood circulation distally to AVF. We did not observe any serious complications (including myocardial infarction, stroke, or death) during the procedure or hospital stay.

Post-procedural course was uneventful except for single case of $17 \mathrm{~mm}$ diameter pseudoaneurysm. It was successfully treated by local thrombin injection. However, up to 30 days post procedure, there were 3 deaths which - according to the opinion of physicians in charge of the patients - were not attributed to the index procedure. Seventeen deaths were noted within the whole observational period, but these events could not be meaningfully related to endovascular procedure (Tab. 3).

Final analysis ( 12 months following the procedure) included 79 patients who remained alive.

Fifty two AVF were functioning properly after 12 months. The probability of the intervention-free survival is shown in Kaplan-Meier estimator curve (Fig. 2). In 20 cases reintervention was performed while 7 others with recurrence of fistula dysfunction did not agree for reintervention (66\% of proper fistula function without reintervention at I year). Time distribution of reintervention in the follow-up period is shown in Figure 3.

Out of all 20 AVF that were subjects of reinterventions, in 4 of them the result of the initial PTA was already not satisfactory. Considering all patients (including those with successful reintervention), 69 AVF were functioning properly at 12 month (87\%).

There was no correlation between the location of lesion (inflow artery, anastomosis, outflow vein) and initial or long-term PTA results. Similarly, the type of balloon catheter were not related to PTA outcome.

Transfemoral approach allowed full rheological imaging of entire vasculature of limb with AVF and direct visualization of arterial part of AVF. There were no differences in procedural outcomes and patient's safety measures dependent on access site. No puncture site related complications were observed in I year follow-up. AVF thrombosis was associated with significantly higher PTA failure rate as compared to stenosis $(2.6 \%$ vs. 13.1\%; $p=0.003$ ).

Other clinical and demographic factors did not influence PTA results.

\section{Discussion}

The dysfunctions of arteriovenous fistulae in our patients were predominantly caused by stenosis, much less frequently by occlusions. Similar observation was made by other authors [15-17]. 
Table 3. Serious adverse events

\begin{tabular}{|c|c|c|}
\hline & \multicolumn{2}{|c|}{ Serious adverse events } \\
\hline & 30 days & I-12 months \\
\hline Death & 3 & 14 \\
\hline Myocardial infarction & 0 & 5 \\
\hline TIA/stroke & I & 0 \\
\hline Congestive heart failure & 2 & 2 \\
\hline Urgent surgical intervention & 0 & 0 \\
\hline Urgent endovascular intervention & 0 & 0 \\
\hline Pseudoaneurysm & I & 0 \\
\hline \multicolumn{3}{|l|}{ Causes of death $0-12$ months $(n=17)$} \\
\hline Myocardial infartion & \multicolumn{2}{|r|}{4} \\
\hline Stroke & \multicolumn{2}{|r|}{ 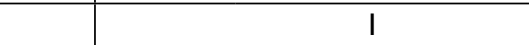 } \\
\hline Congestive heart failure & \multicolumn{2}{|r|}{3} \\
\hline Respiratory failure & \multicolumn{2}{|r|}{ I } \\
\hline Cardiac arrest & \multicolumn{2}{|r|}{ I } \\
\hline Sepsis & \multicolumn{2}{|r|}{ I } \\
\hline Unknown & \multicolumn{2}{|r|}{6} \\
\hline
\end{tabular}

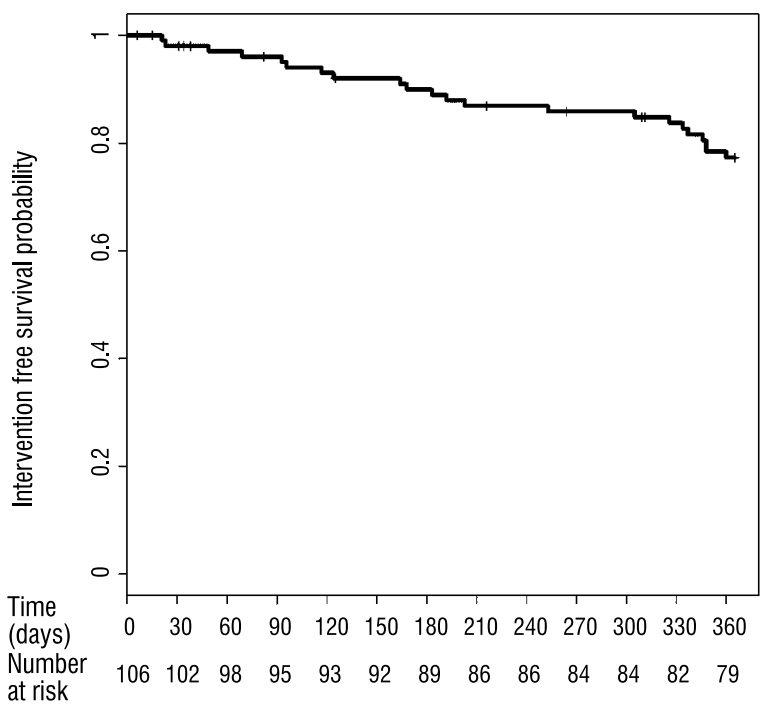

Figure 2. The probability of intervention-free survival (Kaplan-Meier estimator curve)

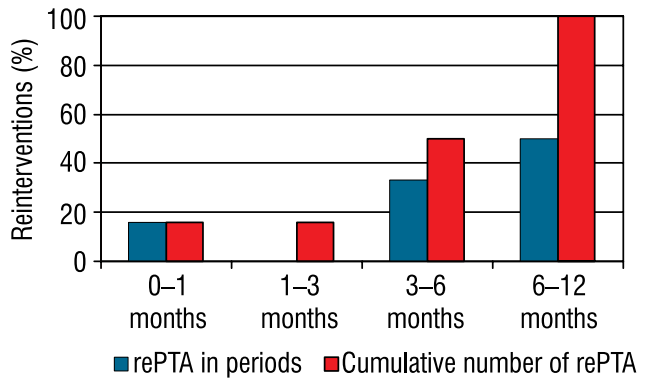

Figure 3. Periodic and cumulative re-intervention rate during I year follow-up
Pathologies within arteriovenous fistulae were mainly located in the outflow vessels. The inflow artery was the least frequent location, which also correlates with observation from other studies [2, 15, 16]. Obstructive lesions of AVF require either endovascular or surgical revascularization. Percutaneous angioplasty of dysfunctional fistulae is a safe treatment modality. In our study, similar to that of other authors $[15,17,18]$, periprocedural SAE (serious adverse events) included a single case of iatrogenic pseudoaneurysm effectively treated by thrombin injection. We did not encounter any deterioration of blood flow within the treated arm. The 3 deaths, which occurred up to 30 days after the procedure, were not related to endovascular treatment.

From our observation, technical success of the procedure was achieved in $90.6 \%$ of patients. The observed $66 \%$ of sufficient clinical effects without clinically driven reintervention and $87 \%$ with reintervention in our patients are noteworthy. It is important to emphasize that the majority of studies available in literature that present results of endovascular treatment of dysfunctional AVF do not rely on single endovascular technique. Frequently, PTA is combined with thrombolysis, thromboaspiration or mechanical thromboaspiration. Natário et al. supplemented PTA with thromboaspiration technique reaching primary patency (PP) of $51 \%$ and secondary patency (SP) of $96 \%$ [19]. In other studies, urokinase or r-tPA (recombinant tissue plasminogen activator) were used in combination with PTA. The treatment results were similar to those presented in the studies where only PTA was used: PP $18-70 \%$, SP - 63-81\% [20-23]. 
Vascular access through femoral artery and the use of long sheaths to manage AVF may not be widely utilized, but has clear advantages. It enables easy visualization of all the inflow and outflow vessels, including the subclavian and axillary arteries and safe cannulation of radial artery even in the cases of its atypical (high) origin. What is needed, though, are long shaft catheters and wires. The brachial artery is quite mobile and may prove to be difficult to cannulate. Moreover, pressure that has to be applied on brachial artery in order to obtain hemostasis may jeopardize the function of the fistula itself. The radial artery may originate from proximal brachial or even axillary arteries, so typical brachial puncture may not provide an access to the fistula. On the other hand, retrograde cannulation of the AVF, using an outflow vein as access, may be quite cumbersome as the fistula usually cannot be sufficiently visualized by injecting the dye into the outflow vein. It seems quite advisable to be skillful in gaining access through each of the sites and choose them according to the needs, for example, cannulate the fistula in a retrograde manner when an attempt at antegrade cannulation fails. In such a case, one may benefit from good visualization of the vessels provided by dye injection into arterial sheath when cannulating the fistula from the venous side.

PTA of main outflow vein was supplemented in some studies by coiling of its branches. Hence, the blood flow could be directed from fistula to the main outflow vessel, thus increasing the flow through AVF $[14,18]$. However, the results of PP (59-60\%) and SP (90\%) are comparable to those from other studies where only PTA was used.

In our study, the location of fistula's pathology had no significant impact neither on immediate nor long-term results of endovascular treatment. We observed significantly worse results of endovascular treatment of occluded fistulas compared to those with stenosis. Similar to our conclusion, Manninen et al. noticed no correlation between the location of lesions patency of fistulas. In contrast, they did not confirm any correlation between the type of pathology (stenosis vs occlusion) and PP rates [15]. Raynaud et al. observed significantly higher number of restenosis within the venous part of fistulas [24].

It has been shown that the long-term clinical effects after endovascular treatment of obstructive AVF lesions have improved over the past years, and are parallel with our own observations [17, 19, 25-28]. It may be related to the availability of low profile, high pressure balloons and growing experience of interventional teams.

The outcomes of surgical and endovascular treatment are corresponding to each other [29]. Due to the reasons that there is only a small number of trials comparing these two methods [29] and no available guidelines, it is not possible to make an evidence-based decision regarding the choice of treatment. Frequently, the choice of particular technique is limited to the practiced method in the specific centre.

Endovascular techniques are less invasive than surgery. Moreover, after percutaneous angioplasty, treated fistula can be catheterized sooner and used as an effective hemodialysis access (usually after two weeks). In the case of surgical procedure, this period is significantly longer [30].

There is little evidence to support the mentioned course of pharmacological treatment and it is inconclusive if antiplatelet or anticoagulant therapy should be used in patients after AVF repair. Since appropriate pharmacological therapy can influence immediate and long-term treatment results of AVF dysfunction, this subject should be addressed in further studies.

\section{Conclusions}

The immediate and long-term outcomes of percutaneous transluminal angioplasty of arteriovenous fistulas with currently available techniques and equipment are satisfying.

Percutaneous angioplasty is a safe manner of prolonging patency rate of arteriovenous fistulas in patients requiring permanent hemodialysis.

\section{References}

I. United States Renal Data System (2007) Annual Data Report. Bethesda, MD: U.S. Department of Health and Human Services, Public Health Service, National Institutes of Health.

2. Machado S, Ferreira A, Lucas C et al (2012) Results of endovascular procedures performed in dysfunctional arteriovenous accesses for haemodialysis. Port J Nephrol Hypert; 26: 266-27I.

3. Chazan JA, London MR, Pono LM (1995) Long-term survival of vascular accesses in a large chronic hemodialysis population. Nephron; 69: 228-233.

4. Stevenson KB, Hannah EL, Lowder CA et al (2002) Epidemiology of hemodialysis vascular access infections from longitudinal infection surveillance data: predicting the impact of NKF-DOQI clinical practice guidelines for vascular access. Am J Kidney Dis; 39: 549-555.

5. Swedberg SH, Brown BG, Sigley R et al (1989) Intimal fibromuscular hyperplasia at the venous anastomosis of PTFE grafts in hemodialysis patients. Circulation; 80: 1726-1736.

6. Miller PE, Carlton D, Deierhoi MH et al (2000) Natural history of arteriovenous grafts in hemodialysis patients. Am J Kidney Dis; 36: 68-74.

7. Glanz S, Gordon DH, Butt KM et al (1987) The role of percutaneous angioplasty in the management of chronic hemodialysis fistulas. Ann Surg; 206: 777-781.

8. Tan LXT, May KK, Robless PA (20I I) Outcomes of endovascular intervention for salvage of failing hemodialysis access. Ann Vasc Dis; 4: 87-92. 
9. Marston WA, Criado E, Jaques PF et al (1997) Prospective randomized comparison of surgical versus endovascular management of thrombosed dialysis access grafts. J Vasc Surg; 26: 373-38I.

10. Vascular Access 2006 Work Group (2006) Clinical practice guidelines for vascular access. Am J Kidney Dis; 48 (Suppl I): SI76-S247.

II. Ng LJ, Chen F, Pisoni RL et al (20 I I) Hospitalization risks related to vascular access type among incident US hemodialysis patients. Nephrol Dial Transplant; 26: 3659-3666.

12. Fistula First Catheter Last (FFCL) Workgroup Coalition. End Stage Renal Disease Network Coordinating Centre. Available at: http://www.fistulafirst.org/ Accessed October 28, 2014. Available at: http://www.fistulafirst.org/.

13. Gotch FA, Sargent JA (1985) A mechanistic analysis of the National Cooperative Dialysis Study (NCDS). Kidney Int; 28: 526-534.

14. Basile C, Casino F, Lopez T (1990) Percent reduction in blood urea concentration during dialysis estimates $\mathrm{Kt} / \mathrm{V}$ in simple and accurate way. Am. J. Kidney Dis; 15: 40-45.

15. Manninen HI, Kaukanen E, Mäkinen K (2008) Endovascular salvage of nonmaturing autogenous hemodialysis fistulas: Comparison with endovascular therapy of failing mature fistulas. J Vasc IntervRadiol; 19: 870-876.

16. Ayez N, Fioole B, Aarts RA (20II) Secondary interventions in patients with autologous arteriovenous fistulas strongly improve patency rates. J Vasc Surg; 54: 1095-1099.

17. Cohen A, Korzets A, Neyman H et al (2009) Endovascular interventions of juxta-anastomotic stenoses and thromboses of hemodialysis arteriovenous fistulas. J Vasc Interv Radiology; 20: 66-70.

18. Miller GA, Hwang W, Preddie D (20II) Percutaneous salvage of thrombosed immature arteriovenous fistulas. Seminars in Dialysis; 24: 107-1 I4.

19. Natário A, Turmel-Rodrigues L, Fodil-Cherif M (2010) Endovascular treatment of immature, dysfunctional and thrombosed forearm autogenous ulnar-basilic and radial-basilic fistulas for haemodialysis. Nephrol Dial Transplant; 25: 532-538.
20. Cho SK, Han H, Kim SS (2006) Percutaneous treatment of failed native dialysis fistulas: Use of pulse-spray pharmacomechanical thrombolysis as the primary mode of therapy. Korean J Radiol; 7: 180-186.

21. Turnel-Rodrigues $L$ (2000) Treatment of failed native arteriovenous fistulae for hemodialysis by interventional radiology. Kidney Int; 57: I I24-I I 40.

22. Shatsky JB, Berns JS, Clark TW (2005) Single-center experience with the Arrow-Trerotola percutaneous thrombectomy device in the management of thrombosed native dialysis fistula. J Vasc Interv Radiol; 16: 1605-1611.

23. Liang HL, Pan HB, Chung HM (2002) Restoration of thrombosed Brescia-Cimino dialysis fistulas by using percutaneous transluminal angioplasty. Radiology; 223: 339-344.

24. Raynaud A, Novelli L, Bourquelot P (2009) Low-flow maturation failure of distal accesses: Treatment by angioplasty of forearm arteries.J Vasc Surg; 49: 995-999.

25. Overbosch EH, Pattynama PM, Aarts HJ (1996) Occluded hemodialysis shunts. Dutch multicenter experience with the hydrolyser catheter. Radiology; 20I: 485-488.

26. Haage P, Vorwerk D, Wildberger JE (2000) Percutaneous treatment of thrombosed primary arteriovenous hemodialysis access fistulae. Kidney Int; 57: I I69-I I75.

27. Rajan DK, Clark TW, Simons ME (2002) Procedural success and patency after percutaneous treatment of thrombosed autogenous arteriovenous dialysis fistulas. J Vasc Interv Radiol; 13: $|2||-12| 8$.

28. Ozkan B, Güngör D, Yıldırım UM et al (2013) Endovascular stent placement of juxta-anastomotic stenosis in native arteriovenous fistula after unsuccessful balloon angioplasty. Iran J Radiol; 10: 133-139.

29. Tordoir JHM, Bode AS, Peppelenbosch N (2009) Surgical or endovascular repair of thrombosed dialysis vascular access: Is there any evidence? J Vasc Surg; 50: 953-956.

30. Dapunt O, Feurstein M, Rendl KH (1987) Transluminal angioplasty versus conventional operation in the treatment of haemodialysis fistula stenosis: Result from a 5-year study. $\mathrm{Br}$ J Surg; 74: 1004-1005. 